\title{
Light Spectrum and Decay Constants in Full QCD with Wilson Fermions*
}

\author{
SESAM-Collaboration: U. Glässner ${ }^{\mathrm{a}}$, S. Güsken ${ }^{\mathrm{a}}$, H. Hoeber ${ }^{\mathrm{b}}$, Th. Lippert ${ }^{\mathrm{b}}$, G. Ritzenhöfer ${ }^{\mathrm{b}}$, \\ K. Schilling ${ }^{\mathrm{a}, \mathrm{b}}$, G. Siegert ${ }^{\mathrm{b}}$ and A. Spitz ${ }^{\mathrm{a}}$. \\ ${ }^{a}$ Physics Department, University of Wuppertal, D-42097 Wuppertal, Germany, \\ ${ }^{b}$ HLRZ c/o Forschungszentrum Jülich, D-52425 Jülich, and DESY, D-22603 Hamburg, Germany.
}

We present results from an analysis of the light spectrum and the decay constants $f_{\pi}$ and $f_{V}^{-1}$ in Full QCD with $n_{f}=2$ Wilson fermions at a coupling of $\beta=5.6$ on a $16^{3} \times 32$ lattice.

\section{INTRODUCTION}

This analysis was performed as part of the SESAM project [1] to investigate Sea quark Effects on Spectrum And Matrix-elements. Configurations were generated using the HybridMonte-Carlo algorithm with the standard Wilson action at a coupling of $\beta=5.6$ on a $16^{3} \times 32$ lattice. We work at three different values of the dynamical quark mass: $\kappa_{\text {sea }}=0.1560,0.1570$ and 0.1575 corresponding to $m_{\pi} / m_{\rho}$-ratios of $0.83(1)$, $0.76(1)$ and $0.71(2)$ [2]. Our lightest quark-mass is approximately equal to the strange quark-mass. Up to now we generated about 8000 trajectories of unit-length with a time-step of $\Delta t=0.01$. After measuring the integrated autocorrelation time for the plaquette and the pion-correlator at fixed timeslice [2,3] we decided to use configurations separated by 25 trajectories for the present spectrum analysis. Our sample consists of 100, 160 and 100 configurations for our 3 sea-quark values. Since we aim at a final sample of $3 \times 200$ configurations this talk should be considered as a half-time status report.

Quark propagators for the set of valence $\kappa$ values $\{0.1555,0.1560,0.1565,0.1570,0.1575\}$ were computed using the standard overrelaxed Minimal Residual Algorithm with smeared sources and local as well as smeared sinks. We used the Wuppertal gauge-invariant gaussian smearing method with $N=50$ iterations and $\alpha=4$, fitting the hadron-correlators to singleexponential functions. Throughout this analyis

*talk presented by U. Glässner we neglect correlations in time and in $\kappa$. We hope to present a stable correlated analysis on our final sample.

\section{CHIRAL EXTRAPOLATIONS WITH FIXED SEA-QUARK MASS}

To determine the critical hopping-parameter $\kappa_{c}$ at a given value of $\kappa_{\text {sea }}$ we fit the pseudoscalar masses according to

$M_{P S}^{2}=b m_{q}+c m_{q}^{2}$

with $m_{q}=1 / 2\left(1 / \kappa-1 / \kappa_{c}\right)$ being proportional to the quark mass. We find $c$ to be significantly different from zero: a linear fit leads to a decrease of $\kappa_{c}$ by four standard deviations with respect to the quadratic fit. The resulting values of $\kappa_{c}$ are shown in table 1 together with their statistical errors. To estimate systematic errors on $\kappa_{c}$, we exclude the largest quark-mass from our data and find a change in $\kappa_{c}$ of the order of one standard deviation.

For the chiral extrapolation of vector masses we use two different parametrizations

$$
\begin{aligned}
& M_{V}=m_{\rho}+b m_{q}+c m_{q}^{2} \\
& M_{V}=m_{\rho}+b m_{q}+c m_{q}^{3 / 2},
\end{aligned}
$$

which are motivated by chiral perturbation theory. In contrast to the $\Pi$ extrapolation we find that the values obtained from 2- and 3parameter fits agree within errors; moreover eqs. 2 and 3 yield identical results. In table 11 we quote the values for the $\rho$-masses and the corresponding lattice-spacings as obtained from the 


\begin{tabular}{llllllll}
$\kappa_{\text {sea }}$ & $\kappa_{C}$ & $a M_{\rho}$ & $a_{\rho}^{-1}[\mathrm{GeV}]$ & $M_{N}[\mathrm{GeV}]$ & $M_{\Delta}[\mathrm{GeV}]$ & $f_{\pi}[\mathrm{MeV}]$ & $f_{V}^{-1}$ \\
\hline 0.1560 & $0.16065(8)$ & $0.359(8)$ & $2.14(5)$ & $1.09(7)$ & $1.30(9)$ & $125(9)$ & $0.33(3)$ \\
0.1570 & $0.15987(6)$ & $0.341(8)$ & $2.23(7)$ & $1.14(6)$ & $1.40(10)$ & $101(8)$ & $0.35(2)$ \\
0.1575 & $0.15963(11)$ & $0.316(10)$ & $2.44(8)$ & $1.09(10)$ & $1.22(8)$ & $118(15)$ & $0.30(3)$
\end{tabular}

Table 1

Extrapolation results with fixed sea-quark mass. Errors quoted are purely statistical

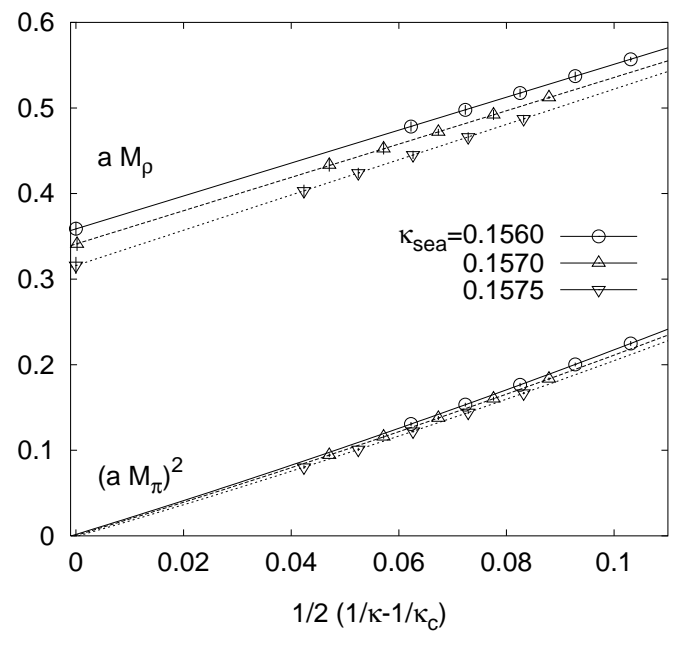

Figure 1. Meson extrapolations with fixed seaquark mass.

2-parameter-fits. The masses are calculated at $\kappa_{c}$ because we find the mass-shifts induced by $\Delta \kappa=\kappa_{c}-\kappa_{\text {light }}$ to be negligible compared to the statistical errors 2 . We will discuss the light quark masses estimated from $\Delta \kappa$ in detail in a fourthcoming publication [2]. Figure 1 illustrates the variation of $m_{\rho}$ with $m_{q}$ and $\kappa_{\text {sea }}$.

The nucleon data (see figure 2) cannot be fitted with $c=0$, but ansätze eqs. 2 and 3 do equally well. The quadratic extrapolation to $\kappa_{c}$ leads to the values $M_{N}$ quoted in table 1 together with the values for $M_{\Delta}$. With the present statistics we do not see any sea-quark dependence of the nucleon mass in physical units.

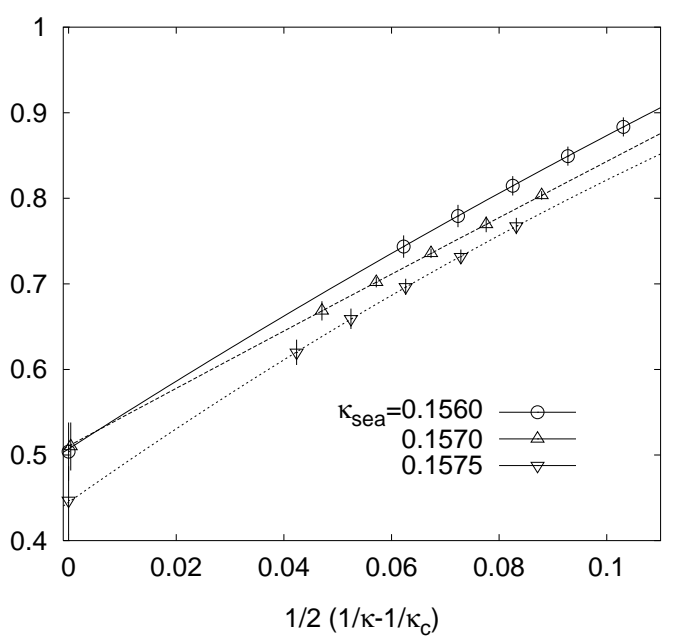

Figure 2. Nucleon extrapolations with fixed seaquark mass.

$\overline{{ }^{2} \text { In fact } \kappa_{c} \text { and } \kappa_{\text {light }} \text { still agree within errors }}$ 

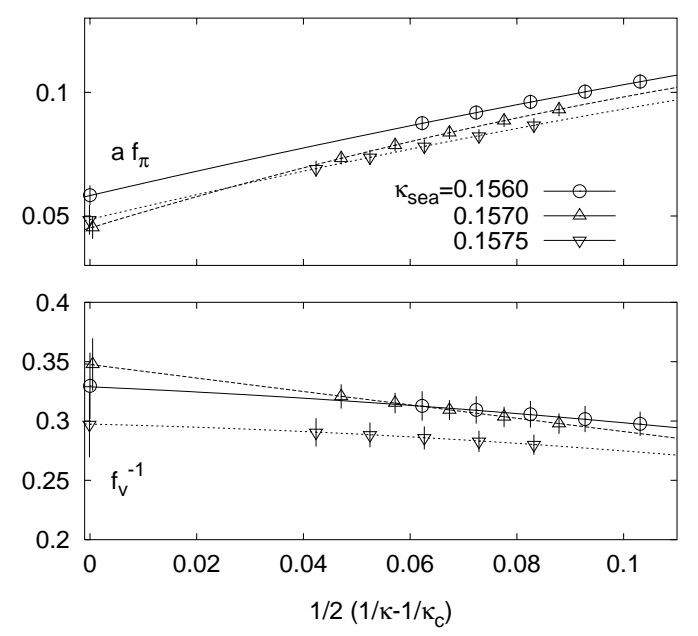

Figure 3. Pseudoscalar and Vector decay constants with fixed sea-quark mass.

We now consider the decay-constants, $f_{\pi}$ and $f_{V}^{-1}$, defined as

$$
\begin{aligned}
<0|A| \pi>Z_{k} Z_{A} & =f_{\pi} m_{\pi} \\
<0\left|V_{i}\right| \rho>Z_{k} Z_{V} & =\epsilon_{i} f_{V}^{-1} m_{\rho}^{2} .
\end{aligned}
$$

$A$ and $V_{i}$ are the local axial and vector currents on the lattice and $Z_{k}, Z_{A}$ and $Z_{V}$ the renormalization constants which connect the expectation values of equation 1 and 5 to the continuum. We determine these renormalization constants using tadpole improved perturbation theory [2, 1 . The $m_{q}$-dependence of the decay-constants is presented in figure 3. Obviously the statistical accuracy is not yet high enough to resolve a $\kappa_{\text {sea }^{-}}$ dependence of $f_{\pi}$ and $f_{V}^{-1}$ at the chiral point (see also table 1).

\section{EXTRAPOLATIONS IN $\kappa_{\text {sea }}$}

A consistent method to extrapolate to zero seaquark mass is to use only the data-points with $\kappa_{\text {sea }}=\kappa_{\text {val }}$ for the extrapolation. In the case of dimensionful observables one might argue about the impact of the varying scale along the trajectory. For this reason we consider only mass-ratios as shown in figure 4 We use linear fits only.

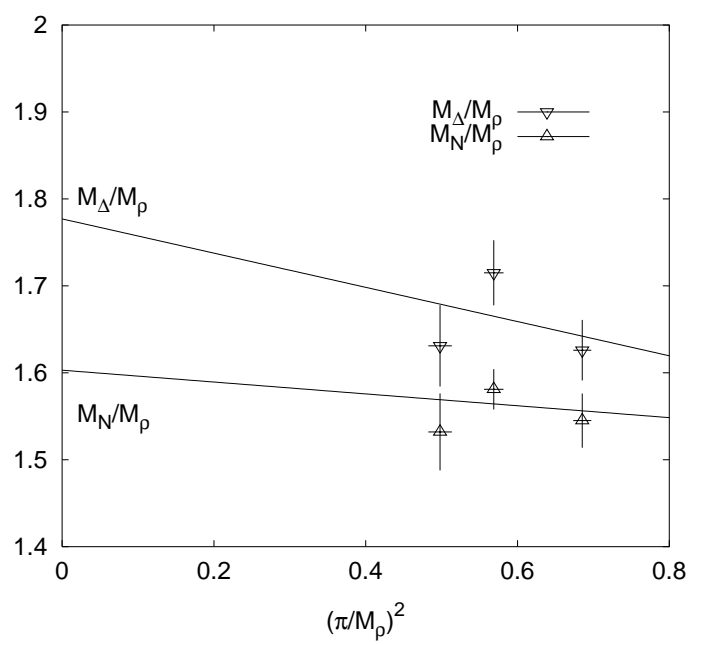

Figure 4. Extrapolations of mass ratios with $\kappa_{\mathrm{sea}}=\kappa_{\mathrm{val}}$.

Table 2

\begin{tabular}{l|l}
\hline$M_{N} / M_{\rho}$ & $1.6(2)$ \\
$M_{\Delta} / M_{\rho}$ & $1.8(2)$ \\
$f_{\pi} / M_{\rho}$ & $0.14(3)$ \\
$f_{V}^{-1}$ & $0.32(6)$ \\
\hline
\end{tabular}

Results from extrapolations in $\kappa_{\text {sea }}$.

The extrapolated values, quoted in table 2, agree (within large errorbars) with those obtained from extrapolations at fixed $\kappa_{\text {sea }}$.

\section{CONCLUSIONS AND OUTLOOK}

On our present statistics, uncorrelated data analyses of $t$ - and $\kappa_{v a l}$ distributions allow for stable chiral extrapolations in valence quark mass, $m_{q}$. We find the $\rho$-meson mass to be be consistent with a linear chiral ansatz, while the remaining quantities considered exhibit nonlinear behaviour in $m_{q} . m_{\rho}$ varies on the level of $15 \%$ across our range of sea quark masses. Baryon masses and decay constants so far indicate a trend but produce no compelling evidence for such variation. 
Chiral extrapolations of our present data in $\kappa_{\text {sea }}$ itself still carry too large uncertainties to be reliable. Better statistics and deeper penetration into the chiral regime are needed to improve on this situation.

\section{REFERENCES}

1. SESAM-Collaboration, Nucl. Phys. B (Proc. Suppl.) 47 (1996) 386.

2. SESAM-Collaboration, in preparation

3. SESAM-Collaboration, Preprint HLRZ 9620 and WUB 96-17, to be published in Phys. Lett. B .

4. G.P. Lepage and P.B. Mackenzie, Phys. Rev. D 48 (1993) 2250. 